\title{
Ultrasonido endoscópico en páncreas
}

\author{
Miguel Angel Ramírez-Luna* \\ Departamento de Endoscopia Gastrointestinal, Instituto Nacional de Ciencias Médicas y Nutrición "Salvador Zubirán”, Ciudad de México, México
}

\section{Resumen}

El ultrasonido endoscópico (USE) ha presentado un desarrollo significativo particularmente para el tratamiento de las enfermedades del páncreas. El drenaje transmural de las colecciones líquidas pancreáticas posterior a pancreatitis aguda, el drenaje del conducto pancreático obstruido en enfermos con pancreatitis crónica o con modificaciones anatómicas posterior a cirugía y el tratamiento local del carcinoma de páncreas guiado por USE representan las principales aplicaciones del USE intervencionista en páncreas.

Palabras clave: Colecciones líquidas pancreáticas. Pseudoquistes pancreáticos. Necrosis pancreática infectada. Pancreatitis crónica. Cáncer de páncreas.

\section{Introducción}

En la pasada Semana Americana de Enfermedades Digestivas realizada en la ciudad de San Diego en Los Estados Unidos de Norteamérica se realizó un Simposio conjunto entre la Asociación Mexicana de Endoscopia Gastrointestinal (AMEG) y la Sociedad Americana de Endoscopia Gastrointestinal (ASGE). Durante este módulo presenté el tema de Ultrasonido Endoscópico Intervencionista en páncreas, que tan lejos podemos llegar, a continuación, describo los aspectos más importantes de esta presentación.

Las indicaciones diagnósticas y especialmente las terapéuticas del USE han crecido significativamente. Actualmente tenemos diferentes opciones para incrementar la exactitud diagnóstica en tumores sólidos y neoplasias quísticas del páncreas tales como la elastografía, el contraste armónico mejorado, las biopsias con minifórceps que pasan a través de una aguja de biopsia y la endomicroscopia láser confocal cuya sonda se puede avanzar también a través de una aguja de biopsia 19G'1 . Especial interés ha sido el desarrollo de varios modelos de agujas que permiten obtener un cilindro de tejido que ofrece las ventajas de permitir analizar la citoarquitectura del tejido lo cual brinda varias ventajas sobre las agujas que solo permitían obtener biopsia por aspiración ${ }^{2}$. Desde el punto de vista terapéutico existe un desarrollo creciente en el tratamiento de las enfermedades pancreáticas y sus complicaciones, evidencia reciente muestra las ventajas de drenar las colecciones líquidas pancreáticas (CLP) especialmente la necrosis encapsulada (NE) e infectada con el uso de prótesis metálicas de aposición luminal que tienen un sistema antimigración, son de diámetro amplio inclusive hasta de $20 \mathrm{~mm}$. y totalmente cubiertas; este mismo diseño especial de prótesis de aposición luminal permiten realizar drenajes coledocoduodenales, drenajes de la vesícula biliar al bulbo duodenal o antro en enfermos con colecistitis aguda y no candidatos a resección quirúrgica así como drenar abscesos intraabdominales posoperatorios y realizar gastroenteroanastomosis en enfermos con obstrucción duodenal

\section{Correspondencia:}


por carcinoma inoperable del páncreas o del duodeno. La terapia de ablación de neoplasias pancreáticas localmente avanzadas es un campo de interés creciente del USE, existe investigación en desarrollo de la ablación con alcohol, terapia fotodinámica, ablación por radiofrecuencia y ablación térmica híbrida ${ }^{3}$.

\section{Drenaje de las colecciones líquidas pancreáticas}

En el pasado el drenaje de las CLP se realizó quirúrgicamente, sin embargo, el advenimiento de las técnicas de invasión mínima ha mostrado ofrecer varias ventajas, particularmente el drenaje guiado por USE.

Varadadarajulu, et al. publicaron un estudio aleatorizado prospectivo que comparó el drenaje de pseudoquistes pancreáticos guiado por USE (PQ-USE) contra la cistogastrostomía quirúrgica (PQ-QX), el éxito del tratamiento fue en 19 enfermos (95\%) del grupo de PQ-USE y en 20 enfermos (100\%) del grupo de PQ-QX sin diferencia significativa. Tampoco hubo diferencias significativas en las complicaciones (PQ-USE: 0\%, PQ-QX:10\%; $p=0.24$ ), en la necesidad de reintervención (PQ-USE: $5 \%$, $P Q-Q X: 5 \% ; p=0.76$ ) ni en la recurrencia (PQ-USE: $0 \%$, $P Q-Q X: 5 \% ; p=0.48)$. Sin embargo, la estancia hospitalaria fue significativamente más corta en el grupo de PQ-USE (mediana de 2 días, rango 1-4) comparado al grupo de $P Q-Q X$ (mediana 6, rango 5-9), $p=<0.001$ y los costos hospitalarios fueron significativamente menores en el grupo de PQ-USE (media: 7,011 USD, DS: 4,171) comparados al grupo de PQ-QX (media 15,052 USD, DS: 10,670$), p=0.003$. Este estudio significó la evidencia más sólida para demostrar las ventajas de realizar el drenaje de los pseudoquistes pancreáticos sintomáticos guiado por USE como un método de invasión mínima que ofrece igual eficacia y seguridad que la cistogastrostomía quirúrgica, pero con dos ventajas sustanciales, menor estancia hospitalaria y menores costos ${ }^{4}$.

De manera similar, al estudio anterior Saúl A, et al. publicamos nuestra experiencia en un estudio retrospectivo en el que comparamos el drenaje de PQ-USE contra el drenaje $P Q-Q X^{5}$. No existieron diferencias significativas en el éxito clínico (PQ-USE: 90.5\%, PQQX: 90.7\%; $p=0.74$ ), complicaciones (PQ-USE: $23.8 \%$, $P Q-Q X: 25.6 \% ; p=0.87$ ), recurrencia (PQ-USE: $9.5 \%$, $P Q-Q X: 4.6 \% ; p=0.59$ ) ni la mortalidad (PQ-USE: $0 \%$, $P Q-Q X: 2.3 \% ; p=0.48)$. Sin embargo, al igual que en el estudio de Varadarajulu, et al. la estancia hospitalaria fue significativamente menor en el grupo de PQUSE (mediana 0 días contra 7 días respectivamente, $\mathrm{p}=<0.0007)$ con costos también significativamente menores (media 3,092 USD contra 7,734 USD, respectivamente, $p=<0.001$ ).

Revisemos ahora la experiencia en el drenaje de la necrosis pancreática infectada guiado por USE.

Van Brunschoy S, et al. publicaron un estudio aleatorizado y multicéntrico de abordaje escalonado quirúrgico o endoscópico para pancreatitis necrotizante infectada ${ }^{6}$. El drenaje guiado por USE de la necrosis pancreática infectada (USE-NPI) inició con la colocación transmural de dos prótesis plásticas doble cola de cochino de 7 French y un catéter nasoquístico de 8.5 French para lavado, si el enfermo no mejoraba clínicamente se realizó necrosectomía endoscópica. El drenaje quirúrgico de invasión mínima (QX-NPI) inició con la colocación de un drenaje percutáneo y si el enfermo no mejoraba clínicamente se realizó necrosectomía retroperitoneal video asistida (VARD). El análisis no demostró diferencias significativas entre los grupo de tratamiento en complicaciones mayores, mortalidad y costo total; sin embargo la insuficiencia cardiovascular de nueva instalación, la frecuencia de fístulas pancreáticas y la estancia hospitalaria fueron significativamente menores en el grupo de drenaje USE-NPI.

Bang, et al. de manera similar al estudio previo compararon el drenaje USE-NPI contra el drenaje QX-NPI'. Los autores no encontraron diferencias significativas entre los grupos de tratamiento en falla orgánica múltiple de nueva instalación, estancia hospitalaria ni en la mortalidad. Sin embargo, el síndrome de respuesta inflamatoria sistémica, la duración del procedimiento, la frecuencia de fístulas pancreáticas y enterales, así como los costos del procedimiento fueron significativamente menores en el grupo de drenaje USE-NPI.

Estudios previos habían demostrado ventajas significativas del drenaje USE-NPI sobre la necrosectomía quirúrgica abierta, tales como menor frecuencia de falla orgánica o empeoramiento de la ya existente, menor frecuencia de complicaciones como fístulas pancreáticas, insuficiencia pancreática endócrina y exocrina, así como hernia incisional. La comparación esperada como lo muestran los dos estudios previos era comparar dos técnicas de invasión mínima, el drenaje USE-NPI contra el drenaje QX-NPI. La conclusión de los dos estudios previos concuerda en que el drenaje USE-NPI sigue teniendo ventajas significativas en reducir costos, complicaciones y estancia hospitalaria.

\section{Drenaje del conducto pancreático}

La pancreatografía retrógrada endoscópica (PRE) con esfinterotomía pancreática y endoterapia intraductal se 
considera el tratamiento de primera elección para los enfermos sintomáticos por obstrucción del conducto pancreático principal. Sin embargo, las fallas técnicas pueden ocurrir hasta en el 10\% de los casos secundaria a estenosis grave del conducto, litiasis intraductal o una papila inaccesible por anatomía modificada ${ }^{8}$. El drenaje del conducto pancreático guiado por USE (CP-USE) es una alternativa mínimamente invasiva, efectiva, segura, técnicamente retadora para el endosonografista y de la cual hay múltiples series en la literatura que informan una resolución de síntomas en $60 \%$ a $80 \%$ de los enfermos, pero desafortunadamente no existen estudios prospectivos controlados ${ }^{9}$.

Chen, et al. publicaron un estudio multicéntrico retrospectivo que comparó el drenaje del CP-USE contra el drenaje por pancreatografía endoscópica retrógrada asistida por enteroscopia (PRE-e) en enfermos con cirugía de Whipple. El éxito técnico (CP-USE: 92.5\%, PRE-e: $20 \%$; $p=<0.001$ ) y clínico (CP-USE: $87.5 \%$, PRE-e: $23 \%$; $p=<0.001$ ) fueron significativamente mejor en el grupo de drenaje CP-USE ${ }^{10}$.

\section{Conclusiones}

Hay un futuro brillante para el USE intervencionista en los años por venir, extensa investigación y varias tecnologías se aplican continuamente a la práctica clínica. El drenaje guiado por USE de las colecciones líquidas pancreáticas y del conducto pancreático obstruido y particularmente el abordaje escalonado endoscópico para drenaje de la necrosis encapsulada e infectada representan alternativas de invasión mínima eficaces y seguras, con menor frecuencia de complicaciones y de falla orgánica así como menor estancia hospitalaria.

\section{Conflicto de intereses}

No existen conflictos de interés. Sin patrocinio de la industria.

\section{Bibliografía}

1. De Lisi S, Giovannini M. Endoscopic ultrasonography: Transition towards the future of gastrointestinal diseases. World J Gastroenterol 2016; 22(5):1779-1786.

2. Coban S, Basar O, Brugge WR. Future Directions for Endoscopic Ultrasound Where Are We Heading? Gastrointest Endoscopy Clin N Am 2017;27:759-772.

3. Cazacu IM, Chavez AA, Saftoiu A, et al. A quarter century of EUS-FNA: Progress, milestones, and future directions. Endosc Ultrasound 2018; 7:141-60.

4. Varadarajulu S, Bang JY, Sutton BS, et al. Equal efficacy of endoscopic and surgical cystogastrostomy for pancreatic pseudocyst drainage in a randomized trial.Gastroenterology 2013;145(3):583-90.

5. Saúl A, Ramírez Luna MA, Chan C, et al. EUS-guided drainage of pancreatic pseudocysts offers similar success and complications compared to surgical treatment but with a lower cost. Surg Endosc 2016,30:1459-1465.

6. Van Brunschoy S, Van Grinsven J, Van Santvoort, et al. Endoscopic or surgical step-up approach for infected necrotising pancreatitis: a multicentre randomised trial. Dutch Pancreatitis Study Group. Lancet 2018;391:51-58.

7. Bang JY, Arnoletti JP, Holt BA, et al. An Endoscopic Transluminal Approach, Compared With Minimally Invasive Surgery, Reduces Complications and Costs for Patients With Necrotizing Pancreatitis. Gastroenterology 2019;156:1027-1040.

8. Tyberg A, Sharaiha RZ, Kedia P, et al. EUS-guided pancreatic drainage for pancreatic strictures after failed ERCP: a multicenter international collaborative study. Gastrointest Endosc 2017;85:164-169.

9. Rimbas M, Largui A. Endoscopic Ultrasonography-Guided Techniques for Accessing and Draining the Biliary System and the Pancreatic Duct. Gastrointest Endoscopy Clin N Am 2017;27:681-705.

10. Chen YI, Levy MJ, Moreels TG, et al. An international multicenter study comparing EUS-guided pancreatic duct drainage with enteroscopy-assisted endoscopic retrograde pancreatography after Whipple surgery. Gastrointest Endosc 2017;85:170-75. 